\title{
Early Enteral Stressors in Newborns Increase Inflammatory Cytokine Expression in a Neonatal Necrotizing Enterocolitis Rat Model
}

\author{
Rebecca Maria Rentea ${ }^{1}$ Scott R. Welak ${ }^{2}$ Katherine Fredrich ${ }^{1}$ Deborah Donohoe ${ }^{3}$ \\ Kirkwood A. Pritchard ${ }^{1,4}$ Keith T. Oldham ${ }^{1,5}$ David M. Gourlay ${ }^{1,5}$ Jennifer L. Liedel ${ }^{6}$
}

\footnotetext{
${ }^{1}$ Department of Surgery, Medical College of Wisconsin, Milwaukee, Wisconsin, United States

2 Department of Neonatology, Medical College of Wisconsin, Milwaukee, Wisconsin, United States

${ }^{3}$ Division of Critical Care, Department of Pediatrics, Medical College of Wisconsin, Milwaukee, Wisconsin, United States

${ }^{4}$ Division of Pediatric Surgery, Medical College of Wisconsin, Milwaukee, Wisconsin, United States

${ }^{5}$ Division of Pediatric Surgery, Children's Hospital of Wisconsin, Medical College of Wisconsin, Milwaukee, Wisconsin, United States

6 Divisions of Neonatology and Critical Care, Children's Hospital of Wisconsin, Medical College of Wisconsin, Milwaukee, Wisconsin, United States
}

Eur J Pediatr Surg 2013;23:39-47.

\begin{abstract}
Address for correspondence and reprint requests Rebecca Maria Rentea, MD, Department of Surgery, Medical College of Wisconsin, 870 W. Watertown Plank Road, Milwaukee, WI 53226, United States (e-mail: Rebeccarentea@gmail.com; rrentea@mcw.edu).
\end{abstract}

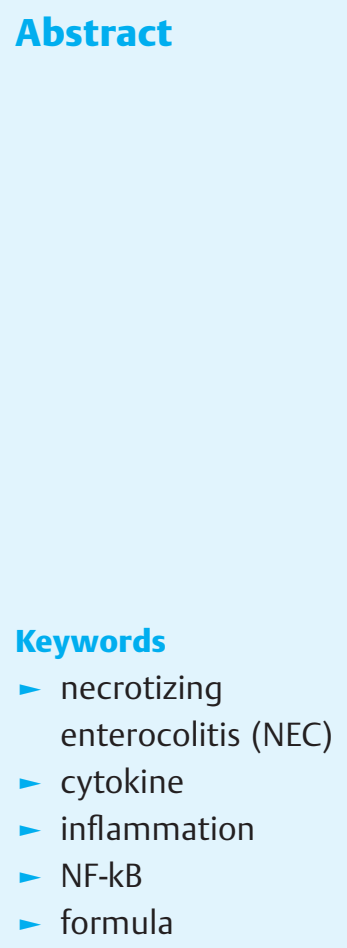

Introduction Inflammation in the premature intestine is a key factor that leads to the development of necrotizing enterocolitis (NEC). Activation of nuclear factor kappa B (NF-KB) and subsequent inflammation increases the severity of NEC. The aim of this study was to investigate the early temporal expression of inflammatory markers and activation of NF-KB in a neonatal rat model of NEC.

Methods Pre- and full-term newborn Sprague-Dawley rat pups were sacrificed at birth, $1.5,4,8$, and 24 hours after receiving their first feed. Control pups were vaginally delivered and mother fed; NEC was induced by a combination of gavage feeding formula, hypoxia, and enteral lipopolysaccharide (LPS); and formula fed pups were fed every 4 hours with infant formula. Ileal tissue was collected for immunohistochemistry, real-time polymerase chain reaction (RT-PCR) and enzyme-linked immunosorbent assay. Serum was collected for cytokine content. Fold change of expression of inducible nitric oxide synthase (iNOS), interleukin (IL)-1 $\beta$, IL-6, tumor necrosis factor- $\alpha$ (TNF- $\alpha$ ), IL-10, NFкB p65, and IкB $\alpha$ used RT-PCR. Data were analyzed by paired two-tailed $t$ test, expressed as mean \pm standard error of the mean, and $p \leq 0.05$ considered significant.

Results No histologic injury was evident in ileal sections. At $1.5 \mathrm{~h}$, iNOS expression increased twofold over control in NEC pups (2.1 vs. 1.0, $p \leq 0.05)$ and remained elevated at $24 \mathrm{~h}(0.7$ vs. 9.4, $p \leq 0.05)$. IL-1 $\beta$ and IL-6 reached a peak at $24 \mathrm{~h}$ in NEC tissue compared with control. IL-10 expression rose in NEC pups after $4 \mathrm{~h}$ of insult and remained elevated in formula and NEC stressed pups. Coincident with an increase in p65 (c) 2013 Georg Thieme Verlag KG Stuttgart · New York
DOI http://dx.doi.org/ $10.1055 / \mathrm{s}-0032-1329704$. ISSN 0939-7248. 
translocation into the nucleus and a reduction of IKB $\alpha$ detected in the cytoplasm, increased transcription of IKB $\alpha$ occurs.

Conclusion These findings suggest that NF-KB activation initiates inflammation early in the course of NEC resulting in increased proinflammatory protein expression, underscoring the importance of the inflammatory response in this NEC model, which precedes evidence of histological injury.

\section{Introduction}

Necrotizing enterocolitis (NEC) is a multifactorial disease of premature infants that involves the gastrointestinal tract. ${ }^{1}$ Occurring in 1 to $10 \%$ of admissions to the neonatal intensive care unit, NEC has an overall mortality rate that ranges from $10 \%$ in mild disease to $100 \%$ in those more severely affected. ${ }^{2}$ NEC survivors may suffer from life-long complications, which include short-bowel syndrome, sepsis, obstruction, malabsorption, and malnutrition. ${ }^{3}$ Risk factors for NEC are numerous and include prematurity, formula feeding, and bacterial colonization of the intestine with nosocomial organisms. Although many risk factors are recognized, the exact etiology and mechanism have not been elucidated.

There are many differences between the immature and mature intestine, which make it more susceptible to injury. In the intrauterine environment, intestinal epithelial cells are bathed in sterile amniotic fluid containing important proteins for maturation. Unlike the mature enterocytes, after preterm delivery, immature neonatal cells are hyperresponsive to stimulation. There may be a robust inflammatory response and altered immune response following exposure to substances and pathogens within the intestinal lumen, inciting a cascade of events that underlies the pathogenesis of NEC. One mediator of inflammation, which plays a large role, is nuclear factor kappa B (NF-KB). Under normal conditions, NF-KB is bound to an inhibitor in the cytoplasm and remains inactive. However, when IKB is phosphorylated, IKB disassociates from the NF- $\mathrm{KB} / \mathrm{IKB}$ is degraded, freeing NF-KB to move into the nucleus to begin transcription of inflammatory proteins. The patterns of expression of cytokines and other inflammatory proteins that occur in NEC impact long-term clinical and neurologic outcomes. ${ }^{4,5}$

The foci of investigations into NEC have largely concentrated on the days that follow initiation of feeding and the evaluation of histologic findings of intestinal injury. Liu et al has demonstrated that activation of intestinal Toll-like receptors and secretion of cytokines in a rat model of NEC precede the histologic injury evident at 3 days. ${ }^{6}$ Within 8 hours of transition from prolonged parenteral nutrition to enteral formula, inflammatory cytokine and brush border enzyme activity transcription change. ${ }^{7}$ These findings suggest that the processes leading to histologic injury may occur at the first formula feed.

Cell culture models utilizing immature enterocytes have also suggested that activation of NF-кB occurs very early and, in turn, regulates interleukin (IL)-6 and IL-8 expression and secretion. Immature intestinal epithelial cells have increased NF-KB activity associated with augmented deoxyribonucleic acid (DNA) binding and subsequent rapid transcription of inflammatory cytokines in response to endogenous tumor necrosis factor- $\alpha(\mathrm{TNF}-\alpha)$. Further downstream, NF-KB regulates nitric oxide (NO) production via its effect on proinflammatory cytokine production. ${ }^{8}$ Moreover, these investigations have suggested that immaturity of ІкВ $\alpha$ (a subunit of the IкB complex) may also have a large role. Heightened NF-KB sensitivity in association with lower baseline expression and more rapid degradation of IKB $\alpha$ contribute to the exaggerated inflammatory response seen in the immature cell. ${ }^{9,10}$ Thus, we hypothesized that the inflammatory response induced by feeding in the gut precedes the later NEC changes known to occur within 72 to 96 hours in the rat NEC model. Little is known about the temporal sequence of events in which enteral feeding and bacterial colonization lead to decreased intestinal defenses and predispose infants to NEC.

Provision of formula to preterm neonates in place of or to supplement and/or fortify maternal milk is standard practice. However, NF-кB and ІкВ $\alpha$ expression do change within 24 hours of formula feeding in an animal model. ${ }^{11}$ We believe that formula feeding combined with the hyperresponse of immature neonatal intestinal epithelium facilitates inflammatory and injurious signals before the development of obvious histologic evidence of NEC. Therefore, we use a time course model to demonstrate the injurious early effect of formula feeding within the first 24 hours after premature birth in a neonatal rat model.

\section{Materials and Methods}

\section{Animal Model}

Animal experiments were approved by the Institutional Animal Care and Use Committee, Medical College of Wisconsin. Control litters of full-term Sprague-Dawley rat pups (Harlan Laboratories, Madison, Wisconsin, United States) were spontaneously delivered and dam fed. Two experimental groups of NEC stressed pups were delivered 1-day prematurely via cesarean section and were gavage fed formula every 4 hours or were fed formula with lipopolysaccharide (LPS), every 4 hours, with three hypoxic episodes per day to simulate stressors associated with NEC. ${ }^{12,13}$ Neonatal rats were fed a base formula of Similac infant formula (4 g; Abbott Laboratories, Columbus, Ohio, United States) in Esbilac canine milk replacement (20 mL; PetAg Inc., Hampshire, Illinois, United States). Formula was supplemented with $2 \mathrm{mg} / \mathrm{kg}$ LPS (SigmaAldrich, St. Louis, Missouri, United States), per feed in the NEC 
group. Pups were sacrificed after birth and 1.5, 4, 8, 12, and 24 hours after their first enteral feeding. The first gavage feed occurred 3 hours after birth.

The pups were sacrificed using intraperitoneal injections of a ketamine and xylazine mixture. Ileal sections were snap frozen, placed in RNAlater (Qiagen Inc., Valencia, California, United States) and fixed in formalin (Richard-Allan Scientific, Kalamazoo, Michigan, United States). Hematoxylin and eosin staining was performed on 4 - $\mu \mathrm{m}$-thick sections. Tissues were graded using a 0 - to 4-point scale previously described. ${ }^{12}$ Serum was collected and processed as described below.

\section{Protein Quantification}

Ileal tissue from neonatal rat pups was cut into small pieces and homogenized in 4-morpholinopropanesulphonic (MOPS) buffer while on ice. ${ }^{14}$ After centrifugation, the supernatant was removed and used for NF-kB p65 translocation assay. Protein content was quantified using the bicinchoninic acid reagent per the manufacturer's protocol (Pierce Chemical Company, Rockford, Illinois, United States).

\section{Immunohistochemistry Staining}

Terminal ileal intestinal segments were removed and fixed in formalin as described previously. ${ }^{13}$ Paraffin-embedded tissues were cut into 4 - $\mu \mathrm{m}$-thick sections. The sections were deparaffinized at $65^{\circ} \mathrm{C}$, immersed in xylene three times, and hydrated with graduated ethanol. For antigen unmasking, slides were heated in 10-mM sodium citrate buffer ( $\mathrm{pH}$ 6.0). Peroxidase activity was quenched with Dako Peroxidase Block (Dako, Carpinteria, California, United States). The specimens were treated with $5 \%$ bovine serum albumin in Trisbuffered saline with $0.05 \%$ ( $\mathrm{vol} / \mathrm{vol}$ ) Tween 20 for 30 minutes at room temperature followed by overnight incubation with anti-NF-кB p65 antibody (D14E12; Cell Signaling, Danvers, Massachusetts, United States) or antiphospho-ІкB $\alpha$ (112B2; Cell Signaling) at $4^{\circ} \mathrm{C}$. After washing, the sections were incubated with polymer-horseradish peroxidase (HRP) antimouse (Dako, Carpinteria, California, United States) for $30 \mathrm{mi}-$ nutes at room temperature. Positive staining was visualized with 3,3'-diaminobenzidine (DAB) chromogen, and nuclei counterstain was performed with hematoxylin (Sigma Chemical Co., St. Louis, Missouri, United States).

\section{Serum Cytokine Assay}

Blood was allowed to clot on ice for 10 minutes and then centrifuged $(5,000 \mathrm{rpm})$ for 10 minutes at $4^{\circ} \mathrm{C}$. The serum was then transferred to a clean polypropylene tube. To completely remove platelets and precipitates, the samples were centrifuged again at $10,000 \mathrm{rpm}$ for 10 minutes at $4^{\circ} \mathrm{C}$. Serum was collected and diluted $1: 2$ in sample diluent. Sera were assayed for cytokine levels (IL-1 $\beta$, IL-6, IL-10, and TNF- $\alpha$ ) using a magnetic bead-based immunoassay kit (Bio-Rad, Hercules, California, United States). Cytokine assays were performed as described by the manufacturer's protocol. The limit of detection of this assay is $1 \mathrm{pg} / \mathrm{mL}$, intra-assay coefficient of variation is $<10 \%$, interassay coefficient of variation is $<15 \%$, accuracy (percentage recovery) is 80 to
$120 \%$, and cross-reactivity is $<1 \%$. Each reaction in the kit was performed in duplicate, with the experiment repeated three times.

\section{Measurement of Nuclear Translocation of NF-кB p65}

Terminal ileal tissue was collected, and snap frozen and stored at $-80^{\circ} \mathrm{C}$. The relative increase of NF-KB/p65 translocation into the nucleus was measured using an enzymelinked immunosorbent assay (ELISA) according to the manufacturer's protocol (Imgenex, San Diego, California, United States). In brief, the tissue was washed with cold phosphate buffered saline (PBS). The tissue was then processed per the manufacturer's protocol resulting in separate cytoplasmic and nuclear fractions. Utilizing $200 \mu \mathrm{g}$ of protein, the assay was completed without deviation from protocol. The relative ratio of nuclear p65 to total cellular p65 was calculated.

\section{Ribonucleic Acid Extraction and RT-PCR}

Real-time polymerase chain reaction (RT-PCR) assays were performed to quantitate expression of IL-1 $1 \beta$, IL-6, IL-10, TNF$\alpha$, and inducible NO synthase (iNOS) with glyceraldehyde 3-phosphate dehydrogenase used as an internal gene control. Total ribonucleic acid (RNA) was isolated from ileal sections with Qiagen RNeasyMini Kit (Qiagen). RNA concentration and purity were quantified by NanoDrop (SPECTRAmas PLUS; Molecular Devices, Sunnyvale, California, United States). Complementary DNA (cDNA) was synthesized from equal amounts $(2 \mu \mathrm{g})$ of DNase-treated RNA using the iScript cDNA Synthesis kit (BioRad, Hercules, California, United States). RTPCR was performed using ABI Prism 7900HT (Applied Biosystems, Carlsbad, California, United States) with SYBR Green RT-PCR Master Mix (Stratagene, Santa Clara, California, United States). PCR amplification for $95^{\circ} \mathrm{C} \times 3$ minutes, 40 cycles of $95^{\circ} \mathrm{C} \times 20$ seconds, $60^{\circ} \mathrm{C} \times 30$ seconds, and $72^{\circ} \mathrm{C} \times 30$ seconds were used. All gene-amplification reactions were performed in triplicate. Primers and probes were designed using National Institutes of Health/National Center for Biotechnology Information (NIH/NCBI) Program Reference Sequence Accession No. iNOS (NM_012611.3), GAPDH (NM_017008.3), IL-1ß (NM_031512.2), IL-6 (NM_012589.1), IL-10 (NM_012854.2), TNF- $\alpha$ (NM_012675.3), NF-KB p65 (NM_199267) exon location 5-6, and ІкB $\alpha$ (NM_001105720) exon location 3-4. RNA expression was compared using the Pfaffl $\Delta \Delta \mathrm{Ct}$ method. ${ }^{15}$ Messenger RNA (mRNA) levels are expressed as relative to control and are presented as mean fold change \pm standard error (SE) of the mean.

\section{Statistical Analysis}

Using SPSS software (version 19; SPSS Inc., Chicago, Illinois, United States), data were analyzed using nonparametric tests since the data have outliers and were skewed. Comparisons were made between the control formula fed and NEC groups as well as each of these groups versus the different time periods. Means and SE are reported for normally distributed data and groups were compared using a $t$ test. Kruskal-Wallis test was used for all groups. Medians and interquartile ranges were reported for skewed data and groups were compared using a Mann-Whitney test. Differences were considered 
statistically significant if the $p$ value was $\leq 0.05$. All tests were conducted as two-tailed tests.

\section{Results}

NF-KB Translocates to the Nucleus following NEC Stress When inflammation is initiated via the NF-кB pathway, IKB $\alpha$ is phosphorylated, dissociated from the cytoplasmic complex, and allowed NF-kB/p65 to move to the nucleus. Translocation of NF-KB/p65 and mRNA expression of NF-KB and $\mathrm{IkB} \alpha$ in the terminal ileum of control, formula fed, and NEC stress exposed pups were evaluated by immunohistochemistry (IHC), ELISA, and RT-PCR. No histologic evidence of NEC injury using the NEC scoring system was present on hematoxylin and eosin staining at 24 hours in the control, formula fed, and NEC pup groups (-Fig. 1). Translocation of NF-KB to the nucleus was detected by IHC utilizing an antibody to the NF-kB/p65 subunit. All animals had detectable p65 staining in the nucleus. However, in NEC pups, there was an increase in nuclear p65 at 4 hours that became less apparent over the following hours. Nuclear staining in the formula fed pups was most abundant at the 24-hour time point (-Fig. 1).

Within the cytoplasm, IкB $\alpha$ sequesters NF-кB until phosphorylation targets it for degradation. Detection of phosphoIKB $\alpha$ within the cytoplasm as a measure of early NF-KB activation used IHC. As shown in -Fig. 2, at 1.5 hours, phospho-ІкB $\alpha$ was present in the cytoplasm of the NEC pups. Small amounts of phospho-ІкВ $\alpha$ was present in the cytoplasm of all animals but were present in greater quantities in formula fed and NEC pups at 4 hours, corresponding to the time point where NF- $\mathrm{KB}$ was also seen in the nucleus (-Fig. 2).

Staining was corroborated with an NF-kB p65 translocation assay. Nuclear and cytoplasmic extracts of terminal ileal tissue were obtained. Nuclear p65 was present at low levels at baseline in control pups. A significant proportion of NF-KB p65 was detected in the nucleus by 4 hours after the first feeding and exposure to stress in the NEC pups, in contrast to mother and formula fed animals ( 0.74 vs. $0.15,0.22$, $p<0.05)$. Control and formula fed pups do not have significant elevations at any time point, although both NF-KB and IкB $\alpha$ continue to increase from birth (-Fig. 3a).

Expression of NF-KB increased in the hours after birth, but did not differ between experimental groups. It was not altered either by administration of feedings or temporally within the first 24 hours after feeding. However, IкB $\alpha$ increased twofold in all formula fed pups over that of controls at 8 hours $(p<0.05)$. It remained persistently elevated and continued to rise in those exposed to NEC stress with a fourfold elevation at 24 hours ( $p<0.05$, - Fig. 3 b).

\section{iNOS Expression Increases following Enteral LPS}

Expression of iNOS, a downstream target of NF-kB, correlated temporally after birth with elevated NF-kB activity. In the

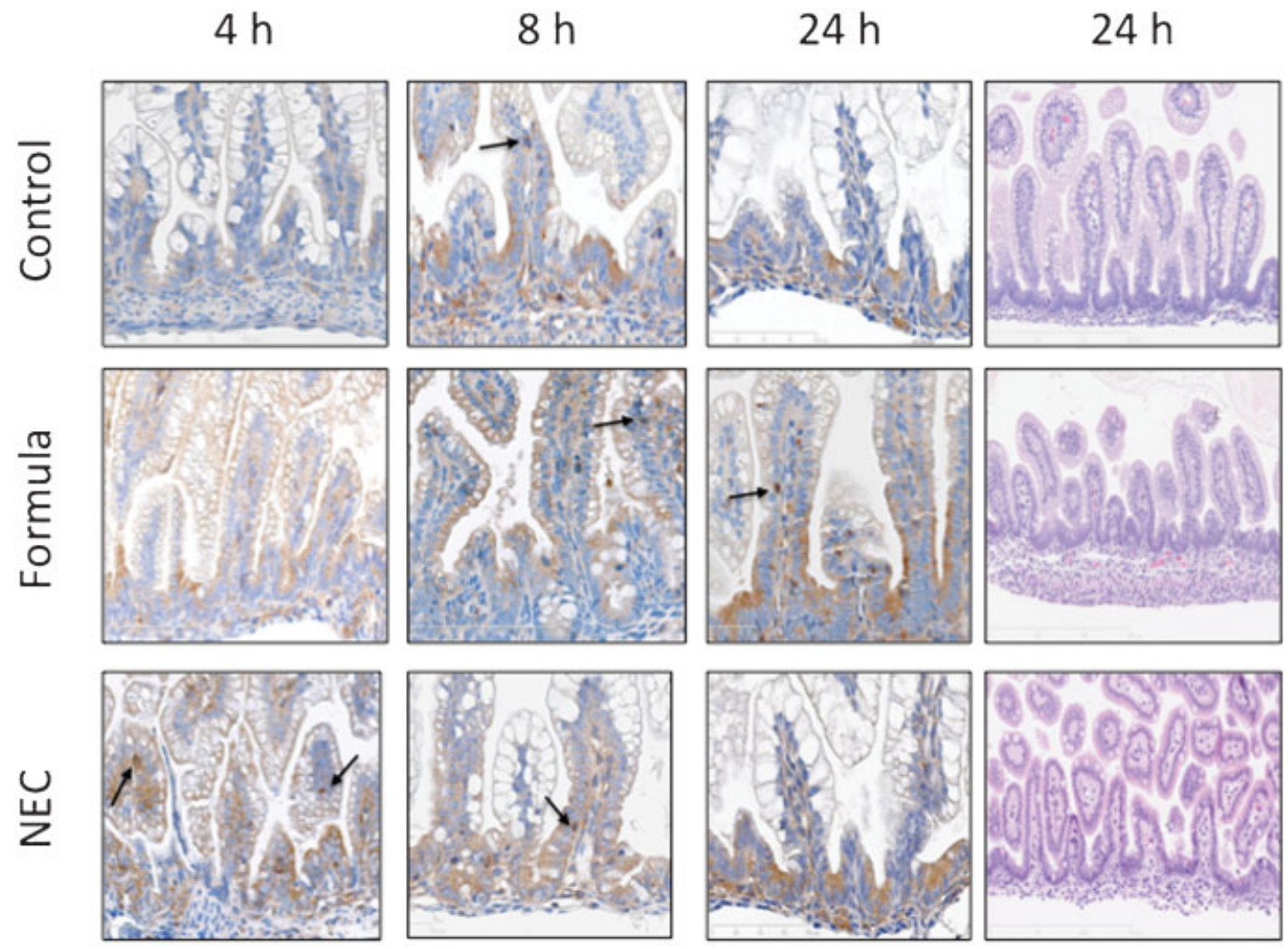

Fig. 1 Immunohistochemical localization of NF-кB p65. Representative pictures of control, formula fed, and NEC stressed terminal ileum at 1.5 to 24 hours after first feeding commenced. NF-kB p65 is localized by antibody staining and is visualized with DAB chromogen and counterstained with hematoxylin. As shown and indicated by arrows, in the formula fed and NEC groups, more NF-kB is localized to the nucleus beginning at 4 hours in the formula fed and NEC stressed groups. Results shown are representative of three separate experiments all with similar results (magnification $\times 200$ ). NEC, necrotizing enterocolitis. 
$1.5 \mathrm{~h}$
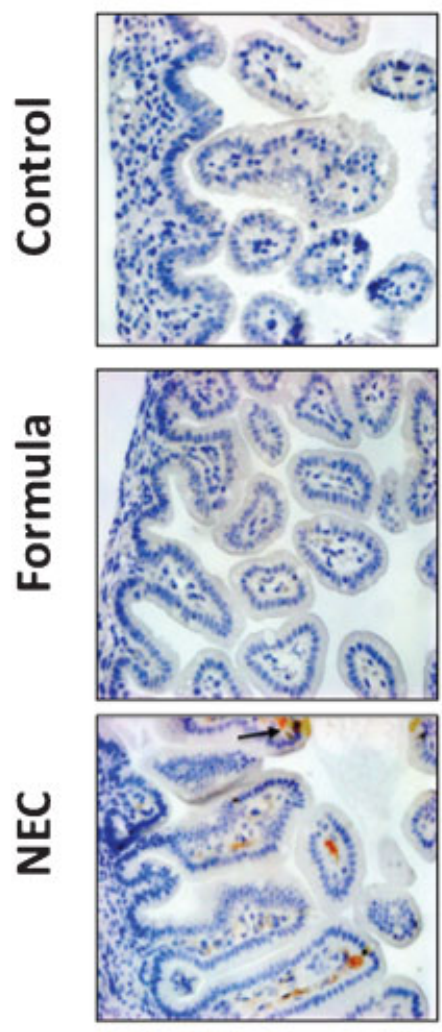

$4 \mathrm{~h}$
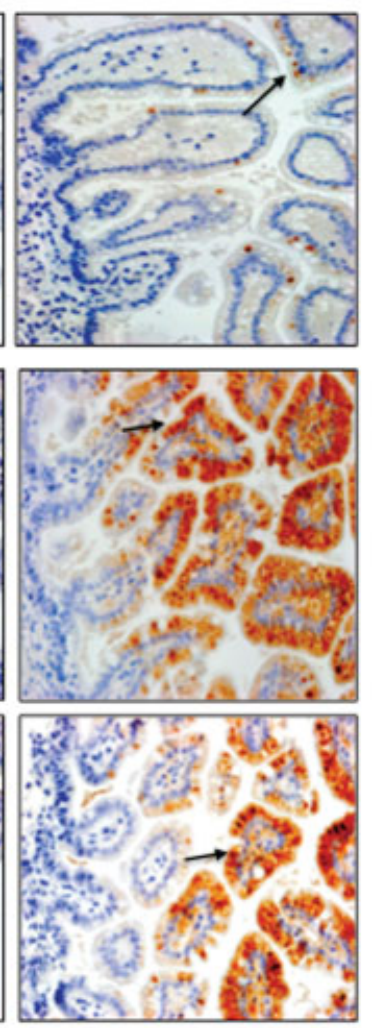

$8 \mathrm{~h}$
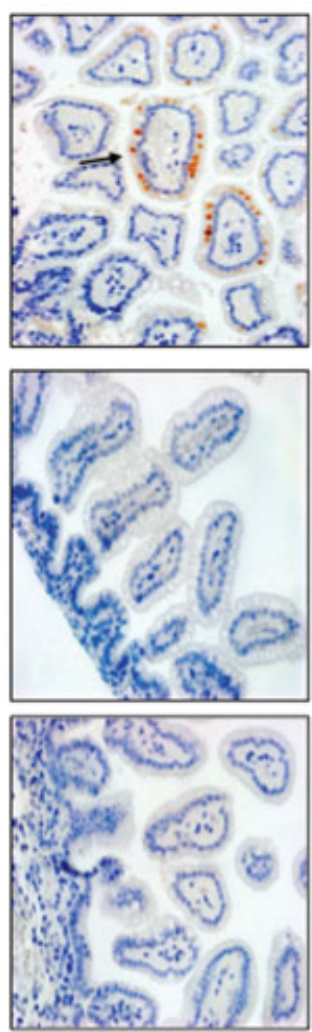

$24 \mathrm{~h}$
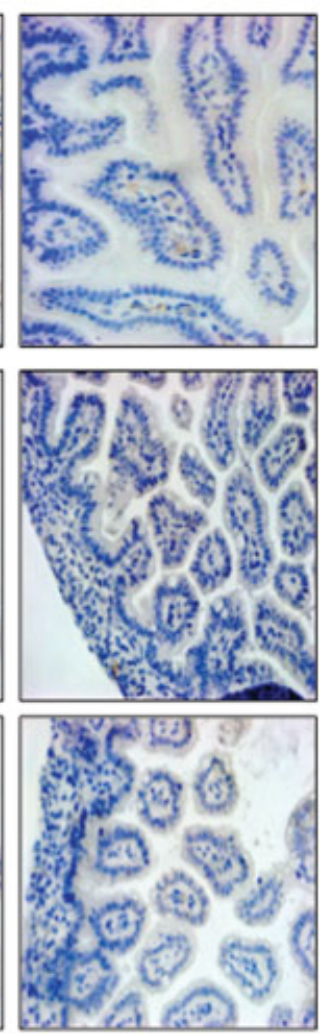

Fig. 2 Immunohistochemical localization of phospho-IкB $\alpha$ subunit of control, formula fed, and NEC stressed terminal ileum at 1.5 to 24 hours after first feeding commenced. Antibody staining was visualized with DAB chromogen and counterstained with hematoxylin. As shown and indicated by arrows, sections have evidence of phospho-IKBa staining beginning at 4 hours in control pups, whereas formula fed and NEC animals have increased staining beginning at 1.5 hours and persisting for several hours. Results shown are representative of three separate experiments (magnification $\times 200)$. NEC, necrotizing enterocolitis.

control rat pup ileum, iNOS expression remained low over time. At birth, there was significantly more iNOS mRNA present in the prematurely delivered pups, but this difference quickly disappeared (1.0 vs. $1.8, p<0.05$, -Fig. 4 ). Its expression was strongly upregulated following the initiation of formula feeding, independent of exposure to stress with LPS and hypoxia at 4 and 8 hours. However, at 24 hours, iNOS expression remained elevated in the NEC pups alone (control 0.76 vs. Eq. 1.44 vs. NEC $2.45, p<0.05)$.

\section{Cytokine Secretion and Expression}

Both hypoxia and LPS may stimulate cytokine secretion. Expression, though, may be induced by NF-KB and there is a delayed rise following p65 translocation. Serum was collected from the pups and TNF- $\alpha$, IL-10, IL-6, and IL-1 $\beta$ levels were quantified. The cytokines, IL-10 and IL-1 $\beta$, did not show any differences between the groups after initiation of feeds, although IL- $1 \beta$ trended upward at 24 hours in the NEC pups $(p<0.05$, -Fig. 5a). IL-10 was detected at relatively high baseline levels in all the groups ( - Fig. $5 \mathrm{~d}$ ) and did change significantly within 24 hours of feeding. Both TNF- $\alpha$ and IL- 6 showed significant elevations over control in the formula fed and NEC stressed pups at 8 hours. Following introduction of feeds, there was a twofold increase in serum TNF- $\alpha$ ( - Fig. $5 \mathrm{c}$, control 978.9 vs. Eq. 2,395.4 vs. NEC 5,094.6 pg/mL, $p<0.05$ ).
Similarly, IL-6 was nearly absent from the control pups at 8 hours but there was a 3,000-fold increase in IL- 6 in the formula group and a 8,000-fold increase in the NEC group ( - Fig. 5b, control 2.2 vs. Eq. 282.9 vs. NEC $8,388.7$ pg/mL, $p<0.05$ ). The level of IL- 6 trended downward between 8 and 24 hours returning to baseline levels in all the groups.

Secretion of cytokines did not directly temporally correlate with mRNA expression. Terminal ileum was subjected to RTPCR analysis. In tissue from the NEC pups, there was an elevation in expression of all cytokines when compared with control at 24 hours (IL-1 $\beta$, IL-6, TNF- $\alpha$, and IL-10, -Fig. $6 a-c)$. Consistent with quantification of secreted levels, TNF- $\alpha$ expression demonstrated a significant elevation at 8 hours in formula fed and stressed pups when compared with control ( 2.45 vs. $10.01, p<0.05$, - Fig. $6 c$ ). At all time points after feeding, there was greater expression of IL-10 in the formula fed and stressed animals. Expression of IL-10 continued to increase with the highest levels at 24 hours (control 2.92 vs. Eq. 6.84 vs. NEC 9.45, $p<0.05$, - Fig. 6d).

\section{Discussion}

These data suggest that early enteral feeding in premature rat pups in combination with known NEC stressors known to incite NEC results in early nuclear translocation of NF-KB. 

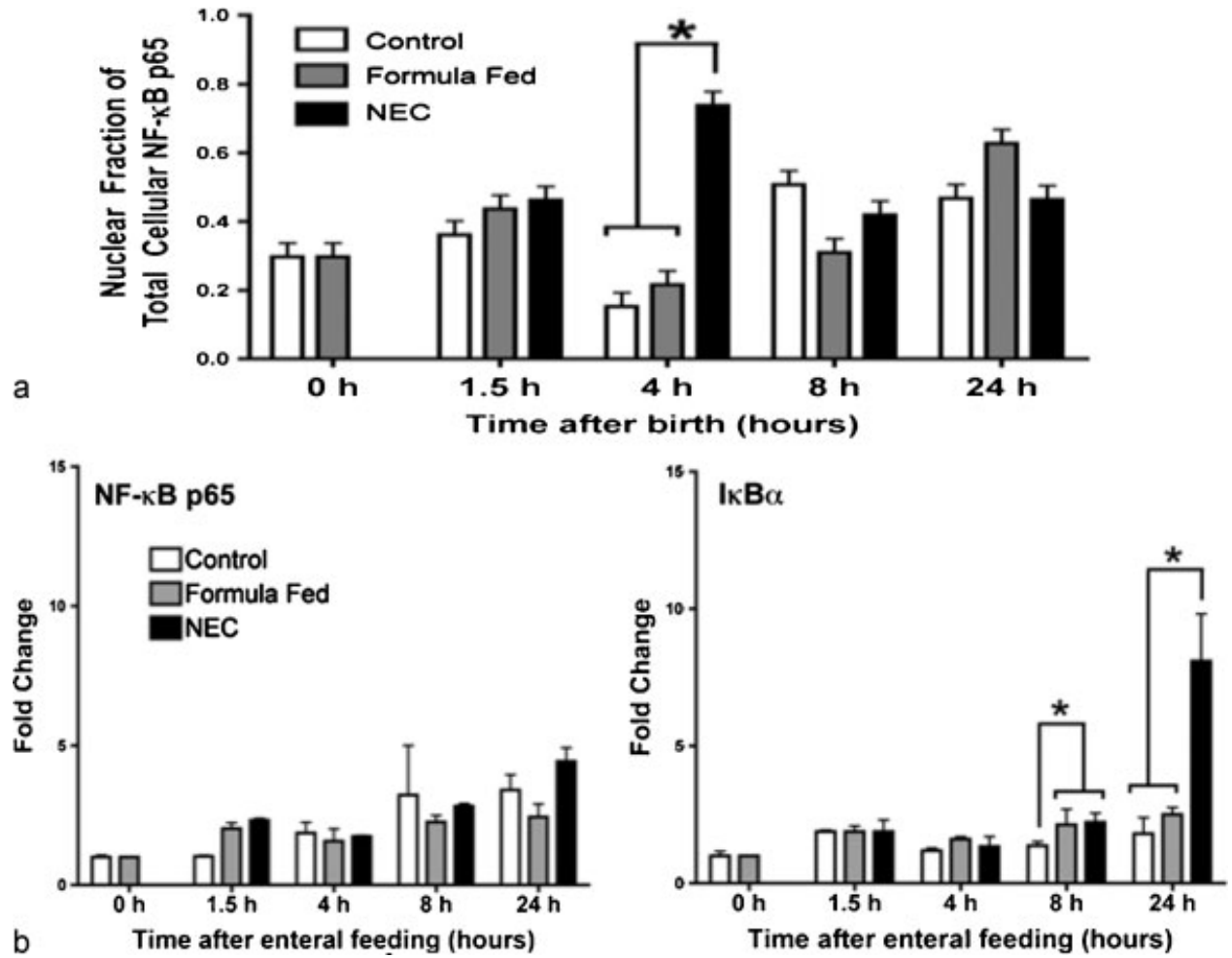

Fig. 3 NF-KB p65 and IкB $\alpha$ gene expression changes in the terminal ileum (a). NF-kB p65 nuclear translocation in the terminal ileum in control, formula fed, and NEC stressed pups. Nuclear extracts were prepared, and the amount of activated NF-KB (in complex with p65) was measured with an ELISA kit with anti-p65 antibodies (b). (means $\pm S E, n=5, p<0.05$ ). ELISA, enzyme-linked immunosorbent assay; NEC, necrotizing enterocolitis; SE, standard errors.

Early enteral stress after birth with formula feeding resulted in the expression of proinflammatory cytokines independent of stress. Markers of inflammation were present within 4 hours of enteral feeding despite the lack of histologic injury.

In this model, the delivery of rat pups at 20 of their 21 - to 22-day gestation equates to an equivalent premature gestational age of a 32 weeks old human premature infant. ${ }^{11}$ Within 1.5 hours of receiving formula, feeds elevation in

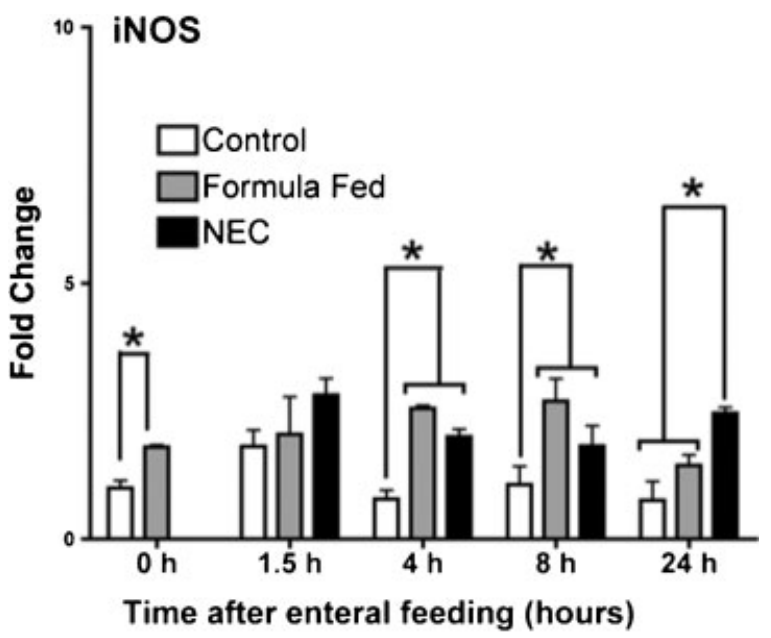

Fig. 4 iNOS gene expression changes in the terminal ileum. iNOS is elevated at birth and continues to increase with enteral stress (means $\pm \mathrm{SE}, n=5, p<0.05$ ). iNOS, inducible nitric oxide synthase; SE, standard errors. serum cytokines were detected in both experimental groups. Following stress with LPS and hypoxia, ІкB $\alpha$ was rapidly phosphorylated, and NF-KB p65 translocation to the nucleus was demonstrated at 1.5 hours with stimulation of transcription of cytokines and iNOS. These findings are consistent with demonstration of early cytokine transcription in the intestine following the transition from parenteral to enteral feeds in preterm piglets. $^{16}$

Numerous mediators are implicated in the inflammatory milieu, which may potentiate NEC. Elevated levels of IL-6, TNF- $\alpha$, and NO products have been demonstrated in septic patients especially neonates with severe NEC. ${ }^{4,17,18}$ Increases in transcription of IL-1 $\beta$, IL-6, and TNF- $\alpha$ as well as iNOS at 24 hours were present in the NEC group receiving LPS. Additionally, these inflammatory mediators (IL-1 $\beta$, IL-6, TNF- $\alpha$, and iNOS) may be released in response to stimulation by both hypoxia and LPS. IL-10 transcription was elevated compared with control pups 1.5 hours after formula feeds in the presence and absence of additional stress with LPS and hypoxia. While IL-10 is an anti-inflammatory cytokine, it is also associated with more severe clinical NEC. ${ }^{4}$ Additionally, IL-10 has been found to be elevated in patients that have gone on to develop sepsis following trauma and thought to result in immunosuppression at high levels. ${ }^{19}$

We found that iNOS increased significantly after both cesarean deliveries as well as following formula feeds. This increase in iNOS returned to control levels within 24 hours in all but the NEC group. iNOS has been demonstrated in both humans as well as in a neonatal rat model to be present with 

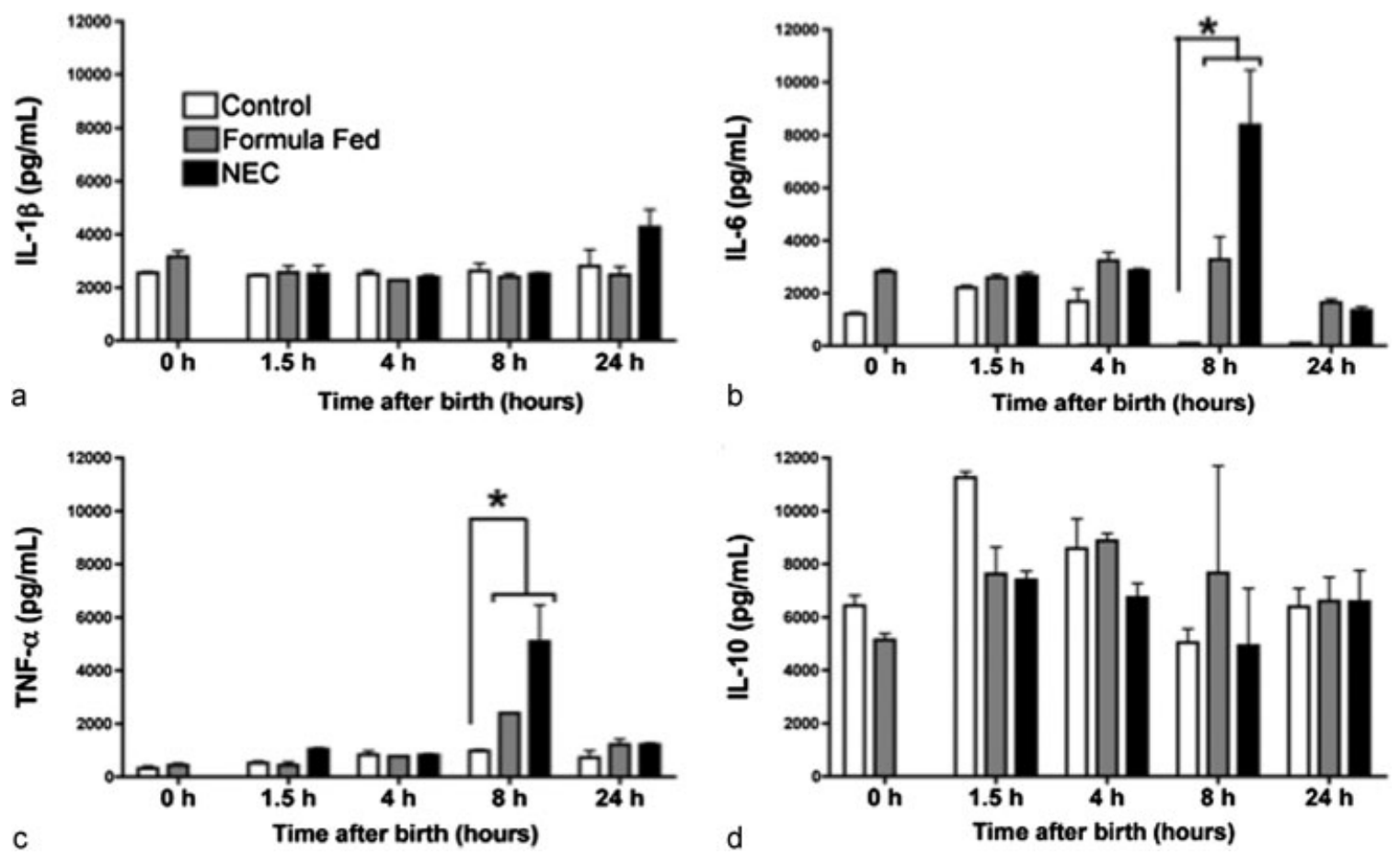

Fig. 5 Inflammatory cytokine gene expression changes in the terminal ileum in control, formula feeding, and NEC stress for 1.5 to 24 hours following the first enteral feeds in neonatal rat pups. Bar graphs show changes in IL-1 $\beta$ (a), IL-6 (b), TNF- $\alpha$ (c), and IL-10 (d) expression in the terminal ileum. Fold changes were analyzed across time points (means $\pm \mathrm{SE}, n=5$ to $7, p<0.05$ ). IL, interleukin; NEC, necrotizing enterocolitis; SE, standard errors; TNF- $\alpha$, tumor necrosis factor- $\alpha$.
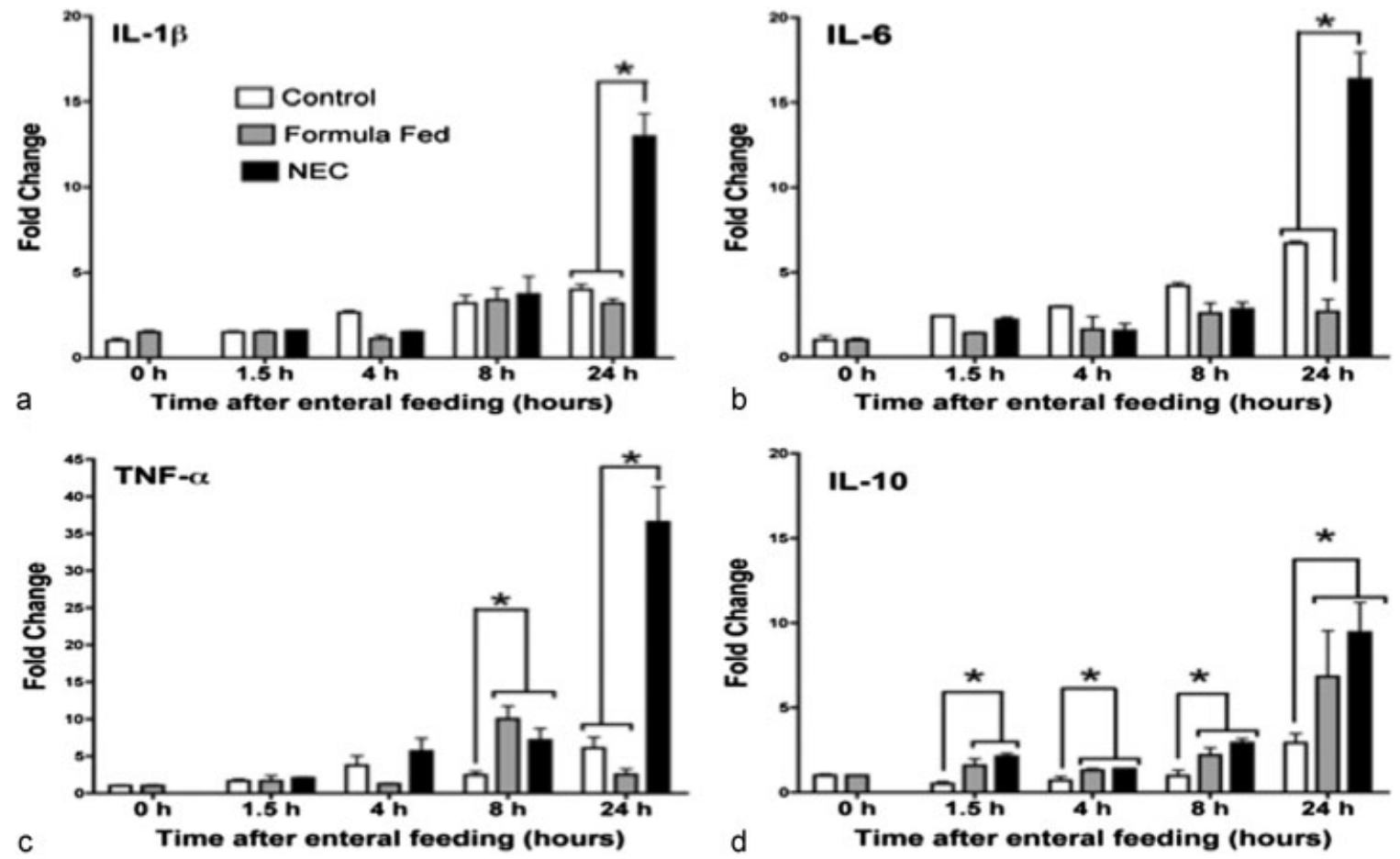

Fig. 6 Systemic inflammatory serum cytokines in control, formula feeding, and NEC stress for 0 to 24 hours following early feeds in neonatal rat pups. Bar graphs show changes in IL-1 $\beta$ (a), IL-6 (b), TNF- $\alpha$ (c), and IL-10 (d). Changes between groups were (means \pm SE, $n=5, p<0.05)$. IL, interleukin; NEC, necrotizing enterocolitis; SE; standard errors; TNF- $\alpha$, tumor necrosis factor- $\alpha$. 
NEC stress but not in uninflamed tissue or mother fed healthy rats. ${ }^{20,21}$ Exposure to LPS in utero increases postnatal intestinal injury in rats. The mechanism of this early injury is mediated by iNOS. Intestinal injury was attenuated and iNOS levels returned to control levels with iNOS inhibition. ${ }^{22}$ Apoptosis as well as the inflammatory response can be incited by iNOS. ${ }^{23}$

Our research findings contribute further evidence that formula feeding can enhance the activation of NF-KB in the intestine, leading to the development of NEC in premature rat pups. Early enteral stress resulted in NF-кB translocation to the nucleus at 4 hours in all formula fed pups. The translocation took place earlier in the NEC group, possibly due to hypoxic induction of NF-kB. ${ }^{24,25}$ Interestingly while NF- $\mathrm{KB}$ is translocated to the nucleus, no increase of NF-kB p65 transcription was observed. In formula fed and NEC stressed pups, at 8 hours, ІкB $\alpha$ expression is upregulated compared with controls, and persists in the NEC stressed ileum at 24 hours. These results reflect an intact negative feedback mechanism in response to inflammation, whereby the cell attempts to turn off or inhibit NF-kB by replacing $\mathrm{IkB} \alpha$, by enhancing transcription of the $\mathrm{IkB} \alpha$ genes. ${ }^{26}$ Previous studies have demonstrated a strong relationship between NF-кB and the pathogenesis of NEC. ${ }^{27}$ The present results solidify a strong early link between the activation of NF-KB and the transcription of cytokines after enteral feeding and stress.

Striking, though, is the difference between all formula fed groups independent of the presence of LPS compared with the control pups. Feeding with maternal milk is known to contain protective factors while formula is devoid of these proteins and factors. Our results raise new questions concerning the early contribution of enteral feeds in the evolution to NEC. What remains to be determined is whether the changes observed can be reversed by the administration of breast milk. In addition, which factors in breast milk are important for the prevention and protection from intestinal inflammation remain to be completely characterized and quantitated. These animals were fed within hours of birth, raising the question of whether time to first feed is important in inflammation in the preterm intestine.

We demonstrate here that within hours of NEC stress, inflammatory profiles and NF-KB translocation to the nucleus take place. Given our findings of early ІкB $\alpha$ and robust inflammatory changes in NEC stressed pups, this leads us to believe that these changes may be clinically applicable in human neonates. Exposure to formula may prime the intestine to have a heightened response to further stressors such as hypoxia and bacterial colonization. These changes occur well before clinical manifestation of NEC in this rat model. This solidifies the concept that formula feeding is not the same as mother's milk. It also stresses the importance of continued investigation to create formula more like mother's milk.

\section{Conclusion}

We have shown that rapid NF-KB translocation and transcription take place in a premature rat model of NEC after enteral feeding is initiated. These results raise the concern of when to commence enteral feeds in premature infants as early inflammatory insults within hours after the first enteral feed may predispose to difficult to revert and may result in the full spectra of NEC.

\section{Acknowledgment}

Research was supported by Children's Research Institute and Clinical and Translation Science Institute (CTSI) of Southeast Wisconsin 1-UL1-RR032973.

\section{Conflict of Interest}

None

\section{References}

1 Lemons JA, Bauer CR, Oh W, et al; NICHD Neonatal Research Network. Very low birth weight outcomes of the National Institute of Child health and human development neonatal research network, January 1995 through December 1996. Pediatrics 2001; 107(1):E1

2 Lin PW, Stoll BJ. Necrotising enterocolitis. Lancet 2006;368 (9543):1271-1283

3 Snyder JK, Barsness K, Munoz R. Gastrointestinal Complications: Necrotizing Enterocolitis, Malrotation, and Protein Losing Enteropathy. New York: Springer; 2010

4 Sharma R, Tepas JJ III, Hudak ML, et al. Neonatal gut barrier and multiple organ failure: role of endotoxin and proinflammatory cytokines in sepsis and necrotizing enterocolitis. J Pediatr Surg 2007;42(3):454-461

5 Markel TA, Crisostomo PR, Wairiuko GM, Pitcher J, Tsai BM, Meldrum DR. Cytokines in necrotizing enterocolitis. Shock 2006; 25(4):329-337

6 Liu Y, Zhu L, Fatheree NY, et al. Changes in intestinal Toll-like receptors and cytokines precede histological injury in a rat model of necrotizing enterocolitis. Am J Physiol Gastrointest Liver Physiol 2009;297(3):G442-G450

7 Siggers RH, Siggers J, Thymann T, Boye M, Sangild PT. Nutritional modulation of the gut microbiota and immune system in preterm neonates susceptible to necrotizing enterocolitis. J Nutr Biochem 2011;22(6):511-521

8 Potoka DA, Nadler EP, Zhou X, Zhang XR, Upperman JS, Ford HR. Inhibition of NF-kappaB by IkappaB prevents cytokine-induced NO production and promotes enterocyte apoptosis in vitro. Shock 2000;14(3):366-373

9 Claud EC, Zhang X, Petrof EO, Sun J. Developmentally regulated tumor necrosis factor-alpha induced nuclear factor-kappaB activation in intestinal epithelium. Am J Physiol Gastrointest Liver Physiol 2007;292(5):G1411-G1419

10 Nanthakumar NN, Fusunyan RD, Sanderson I, Walker WA. Inflammation in the developing human intestine: A possible pathophysiologic contribution to necrotizing enterocolitis. Proc Natl Acad Sci U S A 2000;97(11):6043-6048

11 Okada K, Fujii T, Ohtsuka Y, et al. Overfeeding can cause NEC-like enterocolitis in premature rat pups. Neonatology 2010;97(3): 218-224

12 Whitehouse JS, Riggle KM, Purpi DP, et al. The protective role of intestinal alkaline phosphatase in necrotizing enterocolitis. J Surg Res 2010;163(1):79-85

13 Liedel JL, Guo Y, Yu Y, et al. Mother's milk-induced Hsp70 expression preserves intestinal epithelial barrier function in an immature rat pup model. Pediatr Res 2011;69(5 Pt 1):395-400 
$14 \mathrm{Xu} \mathrm{H}$, Shi Y, Wang J, et al. A heat shock protein 90 binding domain in endothelial nitric-oxide synthase influences enzyme function. J Biol Chem 2007;282(52):37567-37574

15 Pfaffl MW. A new mathematical model for relative quantification in real-time RT-PCR. Nucleic Acids Res 2001;29(9):e45

16 Siggers J, Sangild PT, Jensen TK, et al. Transition from parenteral to enteral nutrition induces immediate diet-dependent gut histological and immunological responses in preterm neonates. Am J Physiol Gastrointest Liver Physiol 2011;301(3):G435-G445

17 Nanthakumar N, Meng D, Goldstein AM, et al. The mechanism of excessive intestinal inflammation in necrotizing enterocolitis: an immature innate immune response. PLoS ONE 2011;6(3):e17776

18 Ochoa JB, Udekwu AO, Billiar TR, et al. Nitrogen oxide levels in patients after trauma and during sepsis. Ann Surg 1991;214(5): 621-626

19 Giannoudis PV, Smith RM, Perry SL, Windsor AJ, Dickson RA, Bellamy MC. Immediate IL-10 expression following major orthopaedic trauma: relationship to anti-inflammatory response and subsequent development of sepsis. Intensive Care Med 2000; 26(8):1076-1081

20 Ford H, Watkins S, Reblock K, Rowe M. The role of inflammatory cytokines and nitric oxide in the pathogenesis of necrotizing enterocolitis. J Pediatr Surg 1997;32(2):275-282
21 Nadler EP, Dickinson E, Knisely A, et al. Expression of inducible nitric oxide synthase and interleukin-12 in experimental necrotizing enterocolitis. J Surg Res 2000;92(1):71-77

22 Giannone PJ, Nankervis CA, Richter JM, Schanbacher BL, Reber KM. Prenatal lipopolysaccharide increases postnatal intestinal injury in a rat model of necrotizing enterocolitis. J Pediatr Gastroenterol Nutr 2009;48(3):276-282

23 Potoka DA, Nadler EP, Upperman JS, Ford HR. Role of nitric oxide and peroxynitrite in gut barrier failure. World J Surg 2002;26(7): 806-811

24 Ahn KS, Aggarwal BB. Transcription factor NF-kappaB: a sensor for smoke and stress signals. Ann N Y Acad Sci 2005;1056:218-233

25 Koong AC, Chen EY, Mivechi NF, Denko NC, Stambrook P, Giaccia AJ. Hypoxic activation of nuclear factor-kappa $B$ is mediated by a Ras and Raf signaling pathway and does not involve MAP kinase (ERK1 or ERK2). Cancer Res 1994;54(20):5273-5279

26 Hoffmann A, Levchenko A, Scott ML, Baltimore D. The IkappaB-NFkappaB signaling module: temporal control and selective gene activation. Science 2002;298(5596):1241-1245

27 De Plaen IG, Liu SX, Tian R, et al. Inhibition of nuclear factor-kappaB ameliorates bowel injury and prolongs survival in a neonatal rat model of necrotizing enterocolitis. Pediatr Res 2007;61(6): 716-721 